\title{
Analysis of Spinal Posture With Three-Dimensional Ultrasonic System in Patients With Ankylosing Spondylitis
}

\author{
Hakan Sercan KURTOĞLU1 ${ }^{1}$, Filiz TUNA ${ }^{2}$, Hakan TUNA ${ }^{3}$, Enis ULUÇAM $^{4}(\mathbb{D}$, \\ Nurettin TAŞTEKIN ${ }^{3}$, Murat BİRTANE ${ }^{3}$ \\ ${ }^{1}$ Department of Physical Medicine and Rehabilitation, Midyat State Hospital, Mardin, Turkey \\ ${ }^{2}$ Department of Physical Therapy and Rehabilitation, Trakya University Faculty of Health Sciences, Edirne, Turkey \\ ${ }^{3}$ Department of Physical Medicine and Rehabilitation, Medical Faculty of Trakya University, Edirne, Turkey \\ ${ }^{4}$ Department of Anatomy, Medical Faculty of Trakya University, Edirne, Turkey
}

\begin{abstract}
Objectives: This study aims to investigate the relationship between postural changes detected in ankylosing spondylitis (AS) vertebrae and clinical and demographic characteristics of patients using a three-dimensional ultrasonic system.

Patients and methods: A total of 44 AS patients ( 36 males, 8 females; mean age $39.1 \pm 9.8$ years; range, 18 to 63 years) and 44 healthy volunteers ( 36 males, 8 females; mean age $38.4 \pm 9.4$ years; range, 18 to 65 years) were included. Clinical, demographic, and laboratory data were recorded. Spinal posture analysis was performed using a Zebris CMS20 three-dimensional ultrasonic system.

Results: Thoracic kyphosis angle was significantly higher in the AS group $\left(45.4 \pm 12.8^{\circ}\right)$ than in the control group $\left(36.9 \pm 7.7^{\circ}\right)(p=0.001)$. Lumbar lordosis angle was significantly lower in the AS group $\left(20.7 \pm 10.6^{\circ}\right)$ than in the control group $\left(28 \pm 8.2^{\circ}\right)(p=0.002)$. There were no significant differences in total trunk inclination or sacral angles $(p>0.05)$. Increase in thoracolumbar length at maximum spinal flexion and decrease in thoracolumbar length at maximum spinal extension were significantly lower in the AS group $(p<0.05)$. A negative correlation was found between the thoracic kyphosis angle and length increase in spine flexure as well as chest expansion. Thoracic kyphosis angle had a statistically significantly positive correlation with age at onset of symptoms and tragus-wall distance $(p<0.05)$.

Conclusion: Significant changes were observed in spinal posture in patients with AS. In addition, significant correlations were found between dynamic postural changes and spinal mobility. We think that postural evaluation and follow-up should be carried out with other parameters. Awareness of AS patients about postural changes should be increased, and proper exercise treatment should be applied.

Keywords: Ankylosing spondylitis; posture; spine.
\end{abstract}

Ankylosing spondylitis (AS) is a disease characterized by chronic inflammation, particularly in the spinal enthesis and surrounding bone. Mechanical stiffness in the affected joints is a reflection of the progressive fusion in adjacent vertebral segments, and decreased range of motion of the joints leads to decreased axial movements. ${ }^{1}$ Posture is a term used to describe a position of the body or the arrangement of body parts relative to one another and analyzed as static and dynamic. Static posture usually refers to the standing or sitting position. Dynamic posture refers to the movement patterns and alignment of a person's body during activity. Postural control is defined as the ability to maintain the body's relationship with the environment, to adjust the alignment of the body under the effects of gravity, and to allow the center of gravity of the

Received: December 25, 2017 Accepted: April 23, 2018 Published online: August 16, 2018

Correspondence: Hakan Sercan Kurtoğlu, MD. Midyat Devlet Hastanesi Fiziksel Tıp ve Rehabilitasyon Kliniği, 47500 Midyat, Mardin, Turkey. Tel: +90 482 - 4621106 e-mail: drhakans@hotmail.com 
body to be properly transferred to the support surface. ${ }^{2}$

Poor posture may cause balance disorders in patients with AS. ${ }^{3}$ Balance disorders in AS patients may also be associated with severe joint deformities. The increased thoracic kyphosis seen in AS patients causes the center of gravity to shift forward and downward. ${ }^{4}$ However, as the disease progresses, these adaptive changes become insufficient and a balance disorder becomes inevitable. With an increased risk of falls, it is easy to develop fractures of the rigid spine. $^{5}$

A combination of non-pharmacological and pharmacological treatments is considered to be the ideal treatment for patients with AS. Non-pharmacological treatment includes patient education and regular exercise. The main purpose of exercise is prevention of flexion and stiffness, and protection and improvement of quality of life and functional capacity. The long-term goal is to provide and maintain a good posture. Exercise therapy may include home-based exercise programs, supervised exercises, group exercises, or spa-based exercises. ${ }^{6}$

An ultrasound-based motion analysis system can be used to assess the spinal posture and range of motion..$^{7-11}$ Test-retest reliability of the neutral kyphosis angle has been demonstrated with a system of this type. ${ }^{12}$ There are only a few reports on the relationship between posture and clinical and demographic characteristics and disease activity in patients with AS.,13,14 Therefore, in this study, we aimed to investigate the relationship between postural changes detected in AS vertebrae and clinical and demographic characteristics of patients using a three-dimensional ultrasonic system.

\section{PATIENTS AND METHODS}

The study was carried out with 88 subjects, 44 with AS (36 males, 8 females; mean age $39.1 \pm 9.8$ years; range, 18 to 63 years) and 44 healthy volunteers (36 males, 8 females; mean age $38.4 \pm 9.4$ years; range, 18 to 65 years) at Medical Faculty of Trakya University between September 2014 and January 2015. The patients with AS were diagnosed according to the modified New York criteria and were followed-up in our rheumatology outpatient clinic. Patients were excluded if they had a history of spinal cord repair surgery; were younger than 18 years or older than 65 years; had neurological diseases (stroke, spinal cord injury, cerebral palsy), mental retardation, limb length inequality, or serious emotional disturbances; or were pregnant. The study protocol was approved by the Medical Faculty of Trakya University Ethics Committee. A written informed consent was obtained from each patient. The study was conducted in accordance with the principles of the Declaration of Helsinki.

Age, sex, height, body weight, and body mass index (BMI) of all subjects were evaluated and recorded. For patients with AS, the age at onset of symptoms, diagnosis age, duration after the diagnosis of the disease, delayed diagnosis (a)

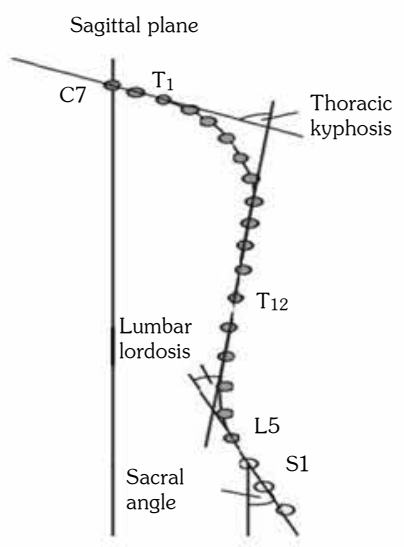

(b)

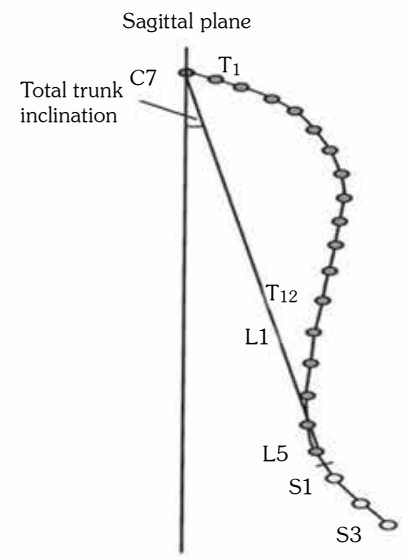

(c)

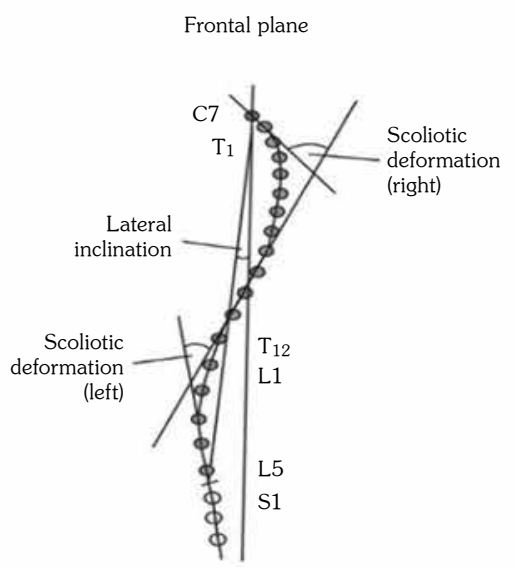

Figure 1. Spinal angles measured in sagittal and frontal planes. 

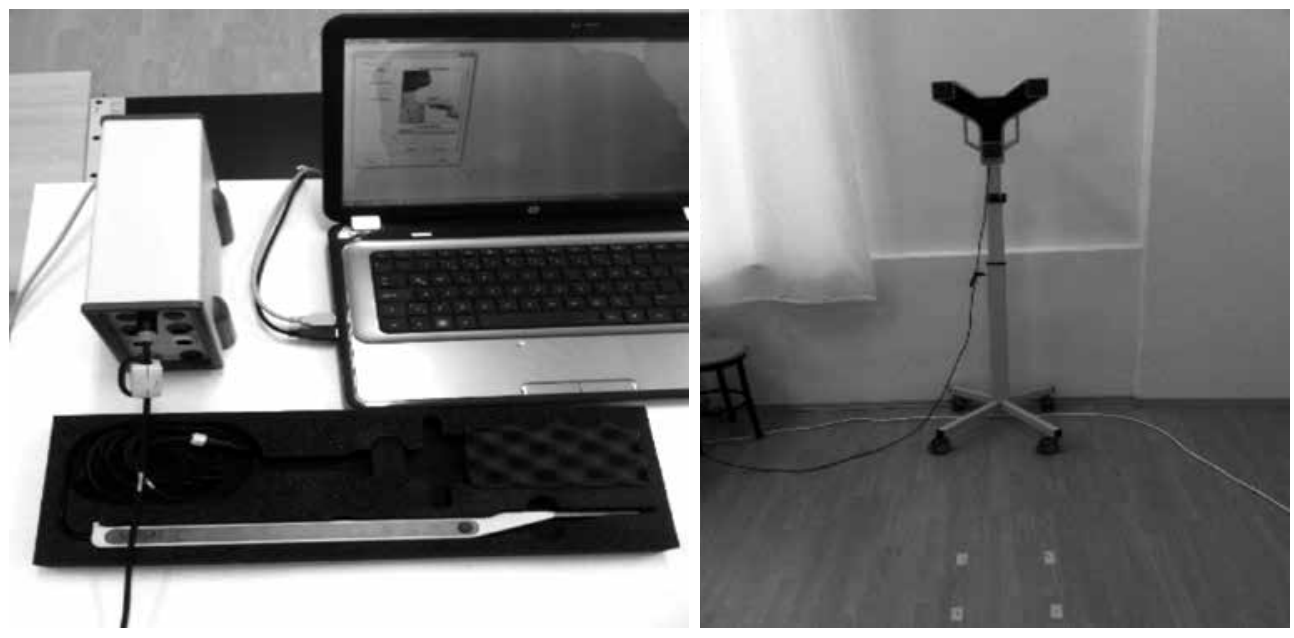

Figure 2. Components of spinal posture evaluation device.

duration, exercise frequency and exercise duration were recorded. The Bath Ankylosing Spondylitis Disease Activity Index (BASDAI), Bath Ankylosing Spondylitis Functional Index (BASFI), and Bath Ankylosing Spondylitis Metrology Index (BASMI) scores were calculated. ${ }^{15}$ Physical examination included measurement of the chin-manubrium sterni distance, occiput-wall distance, fingertip-tofloor distance, and chest expansion. Laboratory analyses included estimation of the C-reactive protein (CRP) level and sedimentation rate. All of these evaluations were performed by the same researcher.

The spinal posture analysis portion of the Zebris CMS20 device (Zebris Medical GmbH, Isny, Germany) was used. With this device, a threedimensional ultrasonic spinal posture analysis was performed as static-dynamic measurements of the spine. The static measurements were performed in the sagittal plane, so that thoracic kyphosis angle, lumbar lordosis angle, sacral angle, and total trunk inclination angle were measured. Scoliosis angle was measured in the frontal plane (Figure 1). ${ }^{8}$ In the dynamic measurements, thoracolumbar spine length was measured $(\mathrm{mm})$ in the neutral position, in flexion, and in extension. The measurement error was $1.96^{\circ}$ and $2.2 \mathrm{~mm}$ for all parameters, and the sensitivity was $0.2 \mathrm{~mm}$ and $0.5^{\circ}$, as defined by the manufacturer. ${ }^{7-9}$ Related studies ${ }^{16,17}$ have shown that this type of ultrasound-based device identifies spinal curvatures at an appropriate level of accuracy and that the intra- and interobserver errors were insignificant. Physical examination and spinal posture analyses were performed in the afternoon so that morning stiffness would not affect the measurements. The data obtained were recorded using WinSpine (Zebris Medical $\mathrm{GmbH}$, Isny, Germany) software.

The components of this measurement system include a measuring device, a sound wave emitting pointer, and software. The measurement device has a height-adjustable lever and is fixed at a distance of $80 \mathrm{~cm}$ to the subject (Figure 2). The pointer contains two sound wave-emitting sources and one button. A square of $25 \times 25 \mathrm{~cm}$ was drawn on the floor, in which the subject stood in a neutral position, and calibration was repeated before each measurement. In the static measurement, when the pointer contacted the reference anatomical points of the subject (acromion, angulus inferior scapulae, spina iliaca anterior superior, spina ilia posterior superior, crista iliaca, between the thoracic vertebra 12 [T12] and first lumbar vertebra [L1], and the spinal column from cervical vertebra $7[\mathrm{C7}]$ to the sacrum), the button was pressed.

In the dynamic measurement, the marked vertebral processes between $\mathrm{C} 7$ and the sacrum at maximum flexion and extension were plotted with a pointer. The software determines the location of the marker in three dimensions, and the measurements were carried out in order. The researcher and the volunteers were not allowed to enter the room with their mobile 
Table 1. Demographic characteristics of patient and control groups

\begin{tabular}{lcccc}
\hline & AS group $(\mathrm{n}=44)$ & & Control group $(\mathrm{n}=44)$ & \\
\cline { 2 - 2 } & Mean $\pm \mathrm{SD}$ & & Mean $\pm \mathrm{SD}$ & $p^{*}$ \\
\hline Age (year) & $39.1 \pm 9.8$ & & $38.4 \pm 9.4$ & 0.733 \\
Height $(\mathrm{cm})$ & $170.7 \pm 8.3$ & & $173.5 \pm 7.6$ & 0.105 \\
Weight $(\mathrm{kg})$ & $75.3 \pm 14.6$ & & $77.6 \pm 14.1$ & 0.447 \\
Body mass index & $25.6 \pm 3.7$ & $25.7 \pm 4.3$ & 0.886 \\
\hline AS: Ankylosing spondylitis; SD: Standard deviation; * Student's t-test. &
\end{tabular}

phones to prevent the measurement results from being affected. ${ }^{8}$ Posture assessment was performed by the same researcher in all cases and conducted three times for each subject, and the average of the three measured values was calculated.

\section{Statistical analysis}

The statistical analysis was performed using IBM SPSS version 20.0 software (IBM Corp., Armonk, NY, USA). Descriptive statistics (mean, standard deviation) were used in the analysis of results. The comparison of age, height, weight, and BMI was performed using the Student t-test. The Mann-Whitney $U$ test was used to compare the spinal curvatures and the length of the spine between the groups. Binary relationships between variables were statistically evaluated using Spearman's correlation analysis. A Spearman's correlation coefficient of $0-0.30$ was considered fair, 0.31-0.50 moderate, 0.51-0.70 good, and 0.71-1.00 excellent. The results were evaluated at the 95\% confidence interval, and a $p$ value of $<0.05$ was considered statistically significant.

\section{RESULTS}

No difference between the two groups was observed for demographic characteristics ( $p>0.05$ ) (Table 1). The mean age at onset of AS symptoms was 25.1 years. The mean delayed diagnosis duration was calculated to be 8.1 years (Table 2).

In the AS group, the degree of thoracic kyphosis was significantly higher $\left(45.4 \pm 12.8^{\circ}\right)$ than in the control group $\left(36.9 \pm 7.7^{\circ}\right)(p=0.001)$. Lumbar lordosis angle was significantly lower than that in the healthy volunteers $(p=0.002)$. No statistically significant difference was observed for total trunk inclination or sacral angles $(p>0.05)$ (Table 3).

There were no statistically significant differences between the groups in terms of the thoracolumbar length values $(p>0.05)$. On the other hand, the increase in thoracolumbar length during maximum spinal flexion and the decrease during maximum spinal extension were

Table 2. Characteristics of patients related to disease

\begin{tabular}{lc}
\hline & $\begin{array}{c}\text { AS group } \\
(\mathrm{n}=44)\end{array}$ \\
\cline { 2 - 2 } & Mean $\pm \mathrm{SD}$ \\
\hline Age at onset of symptoms (year) & $25.1 \pm 9.7$ \\
The duration after the diagnosis of the & $14 \pm 8.9$ \\
disease (year) & $8.1 \pm 7.4$ \\
Delayed diagnosis duration (year) & $3 \pm 2.8$ \\
Exercise frequency (day/week) & $55.4 \pm 83.4$ \\
Exercise duration (minute/week) & $2.3 \pm 1.6$ \\
BASDAI (median) & $2.2 \pm 2.1$ \\
BASFI (median) & $3.3 \pm 1.8$ \\
BASMI (median) & $11.7 \pm 5.2$ \\
Lateral spinal flexion (cm) & $14.1 \pm 5.2$ \\
Tragus-wall distance (cm) & $3.9 \pm 1.8$ \\
Modified Schober measurement (cm) & $92.1 \pm 17.6$ \\
Intermalleolar distance (cm) & $69.7 \pm 23.4$ \\
Cervical rotation (degree) & $3.8 \pm 5.2$ \\
Occiput-wall distance (cm) & $4.1 \pm 2.3$ \\
Chin-manubrium distance (cm) & $3.9 \pm 2.5$ \\
Chest expansion (cm) & $12.2 \pm 11.6$ \\
Fingertip-to-floor distance (cm) & $1.1 \pm 1.9$ \\
C-reactive protein (mg/dL) & $17.9 \pm 14$ \\
Sedimentation (mm/hour) & \\
\hline AS: Ankylosing spondylitis; BASDAI: Bath Ankylosing Spondylitis \\
Disease Activity Index; BASFI: Bath Ankylosing Spondylitis Functional \\
Index; BASMI: Bath Ankylosing Spondylitis Metrology Index. \\
& \\
& \\
&
\end{tabular}


Table 3. Ankylosing spondylitis and control group spinal posture analysis

\begin{tabular}{|c|c|c|c|}
\hline & AS group $(n=44)$ & Control group $(n=44)$ & \\
\hline & Mean \pm SD & Mean \pm SD & $p^{*}$ \\
\hline Thoracic kyphosis angle & $45.4 \pm 12.8$ & $36.9 \pm 7.7$ & 0.001 \\
\hline Lumbar lordosis angle & $20.7 \pm 10.6$ & $28 \pm 8.2$ & 0.002 \\
\hline Total trunk inclination angle & $3.7 \pm 4.5$ & $2.7 \pm 1.6$ & 0.997 \\
\hline Sacral angle & $14.3 \pm 8.6$ & $16 \pm 6.7$ & 0.374 \\
\hline
\end{tabular}

Table 4. Changes of spinal length and their comparison

\begin{tabular}{lccccc}
\hline & AS group $(\mathrm{n}=44)$ & & \multicolumn{2}{c}{ Control group $(\mathrm{n}=44)$} & \\
\cline { 2 - 2 } \cline { 5 - 5 } & Mean $\pm \mathrm{SD}$ & & Mean $\pm \mathrm{SD}$ & $p^{*}$ \\
\hline T-L length (mm) & $491.5 \pm 41.9$ & & $482.7 \pm 28.7$ & 0.174 \\
T-L length increase in flexion (mm) & $92 \pm 41.4$ & & $122.2 \pm 30.4$ & 0.001 \\
T-L length decrease in extension (mm) & $20 \pm 13.2$ & & $34.5 \pm 17.8$ & 0.000 \\
\hline AS: Ankylosing spondylitis; SD: Standard deviation; T-L: Thoracolumbar; * Mann-Whitney U test. & \\
\hline
\end{tabular}

significantly lower in the AS group $(p<0.05)$ (Table 4).

When the thoracic kyphosis angle was compared with clinical and disease activity parameters, there was a significant negative correlation with chest expansion $(p<0.05)$. It was also observed that the thoracic kyphosis angle had a statistically significantly positive correlation with the age at onset of AS symptoms and the tragus-wall distance $(\mathrm{p}<0.05)$. The lumbar lordosis angle had a statistically significantly positive correlation with the modified Schober's test value $(\mathrm{p}<0.05)$ (Table 5).

When the thoracic kyphosis angle was compared with the clinical, laboratory, and disease activity parameters, there was no statistically significant correlation with the lumbar lordosis angle; thoracolumbar spinal length decrease in extension; exercise frequency; exercise duration; duration of the disease; delayed diagnosis duration;

Table 5. Correlation of thoracic kyphosis and lumbar lordosis angle with clinic parameters

\begin{tabular}{|c|c|c|}
\hline & Thoracic kyphosis angle & Lumbar lordosis angle \\
\hline \multicolumn{3}{|c|}{ Age at onset of symptoms } \\
\hline $\mathrm{r}$ & 0.319 & 0.044 \\
\hline $\mathrm{p}$ & 0.035 & 0.776 \\
\hline \multicolumn{3}{|c|}{ T-L length increase in spinal flexion (mm) } \\
\hline $\mathrm{r}$ & -0.462 & 0.060 \\
\hline $\mathrm{p}$ & 0.002 & 0.700 \\
\hline \multicolumn{3}{|c|}{ Chest expansion $(\mathrm{cm})$} \\
\hline $\mathrm{r}$ & -0.367 & 0.183 \\
\hline $\mathrm{p}$ & 0.014 & 0.234 \\
\hline \multicolumn{3}{|c|}{ Tragus-wall distance $(\mathrm{cm})$} \\
\hline r & 0.331 & 0.002 \\
\hline $\mathrm{p}$ & 0.028 & 0.988 \\
\hline \multicolumn{3}{|c|}{ Modified Schober measurement $(\mathrm{cm})$} \\
\hline r & -0.244 & 0.359 \\
\hline $\mathrm{p}$ & 0.110 & 0.017 \\
\hline
\end{tabular}




\begin{tabular}{|c|c|c|}
\hline & $\begin{array}{l}\text { T-L length increase } \\
\text { in flexion }(\mathrm{mm})\end{array}$ & $\begin{array}{l}\text { T-L length decrease } \\
\text { in extension }(\mathrm{mm})\end{array}$ \\
\hline \multicolumn{3}{|c|}{ Thoracic kyphosis angle } \\
\hline r & -0.462 & -0.218 \\
\hline $\mathrm{p}$ & 0.002 & 0.156 \\
\hline \multicolumn{3}{|c|}{ Tragus-wall distance $(\mathrm{cm})$} \\
\hline $\mathrm{r}$ & -0.317 & -0.253 \\
\hline $\mathrm{p}$ & 0.036 & 0.098 \\
\hline \multicolumn{3}{|c|}{ Chin-manubrium distance $(\mathrm{cm})$} \\
\hline r & -0.363 & -0.006 \\
\hline $\mathrm{p}$ & 0.015 & 0.967 \\
\hline \multicolumn{3}{|c|}{ BASMI score } \\
\hline r & -0.341 & -0.084 \\
\hline $\mathrm{p}$ & 0.024 & 0.586 \\
\hline \multicolumn{3}{|c|}{ Exercise duration (minute/week) } \\
\hline r & 0.495 & 0.314 \\
\hline $\mathrm{p}$ & 0.001 & 0.038 \\
\hline \multicolumn{3}{|c|}{ Spinal lateral flexion $(\mathrm{cm})$} \\
\hline $\mathrm{r}$ & 0.409 & 0.134 \\
\hline $\mathrm{p}$ & 0.006 & 0.387 \\
\hline \multicolumn{3}{|c|}{ Modified Schober measurement $(\mathrm{cm})$} \\
\hline r & 0.356 & -0.015 \\
\hline $\mathrm{p}$ & 0.018 & 0.925 \\
\hline \multicolumn{3}{|c|}{ Cervical rotation degree } \\
\hline $\mathrm{r}$ & 0.325 & 0.018 \\
\hline $\mathrm{p}$ & 0.032 & 0.906 \\
\hline
\end{tabular}

mean scores for BASFI, BASDAI, and BASMI; occiput-wall distance; sedimentation rate; or CRP values ( $p>0.05)$.

It was determined that the increase in thoracolumbar length on flexion had a statistically significantly negative correlation with thoracic kyphosis angle, tragus-wall distance, chinmanubrium distance, and BASMI score $(p<0.05)$ (Table 6).

The degree of thoracic kyphosis was compared with the duration after diagnosis, delayed diagnosis duration, and age at onset of symptoms. A statistically significant positive correlation was observed only for the age at onset of AS $(p<0.05)$.

\section{DISCUSSION}

In this study, we aimed to evaluate the spinal posture of AS patients and compare it to that in a control group. Our second aim was to determine the factors that affect the static and dynamic measurements of the spinal column. For the first objective, we performed measurements using an ultrasound-based device. For the second, we examined the parameters that may affect posture in patients with AS.

As a result of the spinal posture analysis with the three-dimensional ultrasonic system, we found that the thoracic kyphosis angle was increased and the lumbar lordosis angle was decreased in patients with AS compared the healthy group. It has been reported that the alterations in the spinal posture of patients with AS decrease lumbar lordosis, increase thoracic kyphosis, and reverse cervical lordosis. ${ }^{3}$ To facilitate diaphragmatic breathing in patients with AS, the abdominal muscles become relaxed. As a result of hip flexion contracture, compensatory knee flexion occurs. ${ }^{3}$

In the literature, there are different methods and results about spinal posture assessment in patients with AS. Postural evaluation of patients with AS is also mostly performed by inclinometer and spinal radiography. ${ }^{4,18}$ As part of the studies evaluating spinopelvic parameters with lateral radiography, Shin et al. ${ }^{14}$ reported a low lumbar 
lordosis angle and a similar thoracic kyphosis angle in patients with AS compared to that in a normal group. However, significant relationships have been reported between clinical parameters and lumbar lordosis in patients with AS. By a similar method, Lee et al. ${ }^{18}$ examined 90 AS patients and 40 healthy volunteers and found no statistically significant differences in the degree of thoracic kyphosis, whereas the lumbar lordosis degree was significantly lower in the AS group. However, there are also some publications that suggest that treatment modalities for AS patients may influence the angle. When the spinal sagittal plan angles of 12 AS patients receiving antitumor necrosis factor treatment and healthy volunteers were evaluated by inclinometer, the thoracic kyphosis angle was higher in the AS group, and the lumbar lordosis angle did not differ. In the same study, there was a significant level of limitation in spinal mobility in the AS group. ${ }^{4}$

The different outcomes observed in different groups of spinal curvatures suggest that in some patients there is no expected change in spinal posture. Although there were significant statistical differences in the spinal curvature between the groups in our study, it is possible to see both very elevated spinal angles and normal angles in patients with AS. By this means, it can be deduced that some patients have a progressive involvement of the spine. The main purpose of posture evaluation is to determine the aspects that are outside of the normal range and try to correct them. Another goal in assessing posture may be to recognize as early as possible those patients who are likely to progress. For this reason, we believe that it is useful to objectively measure and record the posture assessment values of these patients. In addition, to our knowledge, it is not known exactly why the spine posture progresses faster in some patients. In our study, thoracic kyphosis was found to positively correlate with the age at onset of symptoms and negatively correlate with the thoracolumbar length increase in flexion. Therefore, further studies are needed to identify the factors that affect the spinal posture.

The limitation in lumbar spinal mobility in the sagittal and frontal planes is one of the Modified New York criteria. ${ }^{19}$ In our study, the length of the increase in flexion and decrease in extension in the AS group were highly statistically significant, since AS is a disease that limits movement of the spine. We did not find any previous studies of total thoracolumbar length in AS patients. However, the modified Schober's test evaluates spinal mobility in AS patients and is frequently used. In our study, the increase in thoracolumbar length in flexion measured in the AS group was negatively correlated with the thoracic kyphosis angle, tragus-wall distance, chinmanubrium distance, and BASMI score, whereas it was positively correlated with the modified Schober's value, exercise duration, spinal lateral flexion distance, and cervical rotation angle. The thoracolumbar length decrease in extension had a statistically significant positive correlation with weekly exercise duration. We think that dynamic thoracolumbar length measurement, which is an expanded version of the modified Schober's test, may be a parameter to monitor spinal restriction because of the correlation with many values related to spinal mobility.

Posture and balance are closely related concepts and a high occiput-wall distance demonstrates a decreased level of balance; thus, a dynamic balance disorder may occur in AS. There are many body systems used to provide postural stability, such as balance, visual, somatosensorial, and musculoskeletal systems. In patients with AS, the systems related with motor response part of postural control are intact, but the musculoskeletal system is not. ${ }^{2}$ Various results have been reported related to postural stability in patients with AS.1,3,20,21 Dursun et al. $^{5}$ observed that the number of falls experienced by patients with AS was significantly correlated with BASFI and BASMI, whereas there was no significant relationship between BASDAI and the number of falls. As BASFI and BASMI reflect the chronic damage associated with the disease, they may be accepted as more important risk factors when compared with BASDAI. Moreover, Souza et al. ${ }^{22}$ reported that balance in AS patients was worse than in healthy volunteers; furthermore, they stated that there was a positive correlation between pain and balance. Roşu et al. ${ }^{13}$ found that in AS patients, the pain score was higher in patients with hyperkyphosis and scoliosis, whereas the BASFI score was significantly higher in patients with kyphoscoliosis. In our study, there was no statistically significant correlation between the 
thoracic kyphosis angle and BASFI, BASDAI and BASMI mean scores.

In various studies, the positive effects of exercise on the clinical and disease measurements of AS patients have been shown. ${ }^{23-26}$ In our study, it was determined that for AS patients, the weekly exercise duration was positively correlated in a statistically significant level with both the increase in the length of thoracolumbar spine in flexion and decrease of length in extension. The postural alterations observed in AS are one of the most important complaints that affect an AS patient's daily activities. The natural course of the disease distorts the spinal posture and affects the entire body through a chain reaction. The progression of disease has an impact on the postural stability and compensatory strategies. During this period, the main purpose is to maintain the functionality and adaptation in daily life activities. ${ }^{27}$

There are a limited number of articles on spinal posture assessment in patients with AS. In these publications, different methods were used for postural analysis. Spinal posture evaluation in patients with AS is often performed with X-ray. ${ }^{14,18}$ With X-ray, static measurements can be established on the spine while the most important disadvantage is the radiation.

To the best of our knowledge, there is no published literature on AS patients with the device that we used. Our method is radiation free and can assess the spine comprehensively. In addition, the thoracolumbar length can be dynamically measured. Our results showed that serious postural changes might occur in patients with AS, as has been previously reported. We also emphasized the positive effect of exercise in patients with AS by means of thoracolumbar length change, which is a new and different dynamic measurement.

This study has some limitations. We did not compare the posture values measured with the device to X-ray measurements because of the radiation effect, and there were a limited number of subjects. We did not perform intra- and interobserver reliability test. On the other hand, the demographic characteristics of our control group were well-matched to those of the AS group.
In conclusion, significant changes were observed in spinal posture in patients with AS. In addition, significant correlations were found between dynamic postural changes and spinal mobility. We think that postural evaluation and follow-up should be carried out with other parameters. Awareness of AS patients about postural changes should be increased, and proper exercise treatment should be applied.

\section{Declaration of conflicting interests}

The authors declared no conflicts of interest with respect to the authorship and/or publication of this article.

\section{Funding}

The authors received no financial support for the research and/or authorship of this article.

\section{REFERENCES}

1. Vergara ME, O'Shea FD, Inman RD, Gage WH. Postural control is altered in patients with ankylosing spondylitis. Clin Biomech (Bristol, Avon) 2012;27:334-40.

2. Pompeu JE, Romano RS, Pompeu SM, Lima SM. Static and dynamic balance in subjects with ankylosing spondylitis: literature review. Rev Bras Reumatol 2012;52:409-16.

3. Aydog E, Depedibi R, Bal A, Eksioglu E, Unlü E, Cakci A. Dynamic postural balance in ankylosing spondylitis patients. Rheumatology (Oxford) 2006;45:445-8.

4. Sawacha Z, Carraro E, Del Din S, Guiotto A, Bonaldo L, Punzi L, et al. Biomechanical assessment of balance and posture in subjects with ankylosing spondylitis. J Neuroeng Rehabil 2012;9:63.

5. Dursun N, Sarkaya S, Ozdolap S, Dursun E, Zateri C, Altan L, et al. Risk of falls in patients with ankylosing spondylitis. J Clin Rheumatol 2015;21:76-80.

6. Yigit S, Sahin Z, Demir SE, Aytac DH. Homebased exercise therapy in ankylosing spondylitis: short-term prospective study in patients receiving tumor necrosis factor alpha inhibitors. Rheumatol Int 2013;33:71-7.

7. Kowalski IM, Protasiewicz-Faldowska H, Dwornik M, Pierozynski $\mathrm{B}$, Raistenskis J, Kiebzak W. Objective parallel-forms reliability assessment of 3 dimension real time body posture screening tests. BMC Pediatr 2014;14:221.

8. Glista J, Pop T, Weres A, Czenczek-Lewandowska E, Podgórska-Bednarz J, Rykala J, et al. Change in anthropometric parameters of the posture of students of physiotherapy after three years of professional training. Biomed Res Int 2014;2014:719837.

9. Takács M, Rudner E, Kovács A, Orlovits Z, Kiss $\mathrm{RM}$. The assessment of the spinal curvatures in the 
sagittal plane of children using an ultrasound-based motion analysing system. Ann Biomed Eng 2015;43:348-62.

10. Walicka-Cuprys K, Skalska-Izdebska R, Rachwal M, Truszczynska A. Influence of the Weight of a School Backpack on Spinal Curvature in the Sagittal Plane of Seven-Year-Old Children. Biomed Res Int 2015;2015:817913.

11. Mannion AF, Klein GN, Dvorak J, Lanz C. Range of global motion of the cervical spine: intraindividual reliability and the influence of measurement device. Eur Spine J 2000;9:379-85.

12. Fölsch C, Schlögel S, Lakemeier S, Wolf U, Timmesfeld N, Skwara A. Test-retest reliability of 3D ultrasound measurements of the thoracic spine. PM R 2012;4:335-41.

13. Roşu MO, Ancuta $\mathrm{C}$, Iordache $\mathrm{C}$, Chirieac $\mathrm{R}$. Importance of posture assessment in ankylosing spondylitis. Preliminary study. Rev Med Chir Soc Med Nat Iasi 2012;116:780-4.

14. Shin JK, Lee JS, Goh TS, Son SM. Correlation between clinical outcome and spinopelvic parameters in ankylosing spondylitis. Eur Spine J 2014;23:242-7.

15. Sieper J, Rudwaleit M, Baraliakos X, Brandt J, Braun J, Burgos-Vargas R, et al. The Assessment of SpondyloArthritis international Society (ASAS) handbook: a guide to assess spondyloarthritis. Ann Rheum Dis 2009;68:1-44.

16. Ripani M, Di Cesare A, Giombini A, Agnello L, Fagnani F, Pigozzi F. Spinal curvature: comparison of frontal measurements with the Spinal Mouse and radiographic assessment. J Sports Med Phys Fitness 2008;48:488-94.

17. Mannion AF, Knecht K, Balaban G, Dvorak J, Grob D. A new skin-surface device for measuring the curvature and global and segmental ranges of motion of the spine: reliability of measurements and comparison with data reviewed from the literature. Eur Spine J 2004;13:122-36.

18. Lee JS, Suh KT, Kim JI, Goh TS. Analysis of sagittal balance of ankylosing spondylitis using spinopelvic parameters. J Spinal Disord Tech 2014;27:E94-8.
19. van der Linden S, Valkenburg HA, Cats A. Evaluation of diagnostic criteria for ankylosing spondylitis. A proposal for modification of the New York criteria. Arthritis Rheum 1984;27:361-8.

20. Murray HC, Elliott C, Barton SE, Murray A. Do patients with ankylosing spondylitis have poorer balance than normal subjects? Rheumatology (Oxford) 2000;39:497-500.

21. Bot SD, Caspers M, Van Royen BJ, Toussaint HM, Kingma I. Biomechanical analysis of posture in patients with spinal kyphosis due to ankylosing spondylitis: a pilot study. Rheumatology (Oxford) 1999;38:441-3.

22. Souza MCd, Tutiya GdC, Jones A, Júnior IL, Natour J. Assessment of Functional Balance and Quality of life Among Patients with Ankylosing Spondylitis. Rev Bras Reumatol 2008;48:274-7.

23. Martins NA, Furtado GE, Campos MJ, Leitão JC, Filaire E, Ferreira JP. Exercise and ankylosing spondylitis with New York modified criteria: a systematic review of controlled trials with metaanalysis. Acta Reumatol Port 2014;39:298-308.

24. Roşu MO, Topa I, Chirieac R, Ancuta C. Effects of Pilates, McKenzie and Heckscher training on disease activity, spinal motility and pulmonary function in patients with ankylosing spondylitis: a randomized controlled trial. Rheumatol Int 2014;34:367-72.

25. Kasapoglu Aksoy M, Birtane M, Taştekin N, Ekuklu G. The Effectiveness of Structured Group Education on Ankylosing Spondylitis Patients. J Clin Rheumatol 2017;23:138-43.

26. Aytekin E, Caglar NS, Ozgonenel L, Tutun S, Demiryontar DY, Demir SE. Home-based exercise therapy in patients with ankylosing spondylitis: effects on pain, mobility, disease activity, quality of life, and respiratory functions. Clin Rheumatol 2012;31:91-7.

27. Spoorenberg A, van der Heijde $\mathrm{D}$, de Klerk $\mathrm{E}$, Dougados $\mathrm{M}$, de Vlam $\mathrm{K}$, Mielants $\mathrm{H}$, et al. A comparative study of the usefulness of the Bath Ankylosing Spondylitis Functional Index and the Dougados Functional Index in the assessment of ankylosing spondylitis. J Rheumatol 1999;26:961-5. 